\title{
Density and distribution of anionic sites on boar ejaculated and epididymal spermatozoa
}

Accepted: 5 March 2002 / Published online: 23 April 2002

(C) Springer-Verlag 2002

\begin{abstract}
Current knowledge implies that spermatozoa successively acquire negative surface charges as they migrate through the epididymis. Until recently, however, techniques used were not amenable to statistical analysis. In the present study, a novel approach allowing numerical assessment of negative charge labelling was used in order to determine the density and distribution of anionic sites on ejaculated and maturing spermatozoa collected from six regions of the boar epididymis. Labelling was assessed quantitatively for the three morphologically distinct membrane domains on the sperm head. Statistical analysis revealed that labelling density was highest on efferent duct spermatozoa, declined up to the proximal corpus and then increased again. Densities of anionic sites on distal corpus, proximal cauda and ejaculated sperm cells were similar but significantly below the values obtained for efferent duct spermatozoa. All three sperm membrane domains underwent parallel changes. However, the overall density of negative charges on the postacrosomal segment was significantly higher as compared to the acrosomal plasma membrane. These alterations reflect sperm surface modifications through removal and addition of anionic groups. Since charge interactions are considered to play a pivotal role in sperm-egg interactions, these processes should be viewed as an integral part of sperm maturation.
\end{abstract}

Keywords Negative charges $\cdot$ Sperm maturation . Quantitative analysis

M.H. Stoffel ( $)$ A.E. Friess

Department of Veterinary Anatomy,

University of Berne Veterinary School,

Länggass-Strasse 120, 3012 Bern, Switzerland

e-mail: michael.stoffel@ita.unibe.ch

Tel.: +41-31-6312205, Fax: +41-31-6312615

A. Busato

Maurice E. Müller Center for Continuing Education and Documentation in Orthopaedic Surgery, Berne, Switzerland

\section{Introduction}

A number of studies have dealt with the presence of electrical charges at the surface of sperm cells. Methods used included whole cell microelectrophoresis (Bedford 1963; Lopez et al. 1987; Nevo et al. 1961), isoelectric focusing (Moore 1979), labelling with tritiated polycationised ferritin followed by scintillation counts (Toowicharanont and Chulavatnatol 1983), binding of charged beads (Chulavatnatol et al. 1978) and visualisation of anionic sites in transmission electron microscopy (TEM) after direct labelling with cationic probes such as colloidal iron hydroxide, ruthenium red and cationised ferritin (Danon et al. 1972; Delgado et al. 1990; Fléchon 1975; Lopez et al. 1987; Yanagimachi et al. 1972). As concluded from electrophoretic mobility and qualitative judgment of sperm labelling density in TEM, spermatozoa were thought to successively acquire acidic groups as they migrate through the epididymis (Bedford 1963; Lopez et al. 1987; Toowicharanont and Chulavatnatol 1983). To date, however, this contention has defied statistical verification since methods allowing quantitative assessment of labelling densities as counts per unit cell surface area have been lacking. In order to remedy this situation, we recently established a novel method allowing the detection of anionic sites at the cell surface by scanning electron microscopy (SEM) after cationised colloidal gold labelling (Stoffel and Friess 2002). This approach now provides the possibility to assess the occurrence of negative charges numerically. The present study, therefore, was designed to revisit the density and distribution of anionic sites on ejaculated and maturing spermatozoa in the boar. Statistical analysis revealed that the number of negative charges per surface area does not increase continuously on the sperm heads during epididymal transit but rather is highest on efferent duct spermatozoa, declines up to the proximal corpus and then rises again. Density of anionic sites on cauda and ejaculated sperm cells thus return to the level detected on proximal caput spermatozoa. Mechanisms underlying these sperm surface modifications are removal and addition of 
anionic groups such as phosphates and sialic acid residues. These processes are likely to represent an important facet of sperm maturation since charge interactions are considered to play a pivotal role in cell-cell interactions in general and fertilisation in particular (Iqbal and Hunter 1995; Kim et al. 1997; Philip et al. 1997)

\section{Materials and methods}

Materials

Epididymal sperm

Immediately after castration, testes with epididymides from mature Large White boars (approximately $120 \mathrm{~kg}$ body weight) were perfused with PBS $\left(2.9 \mathrm{mM} \mathrm{NaH} \mathrm{PO}_{4}, 7.1 \mathrm{mM} \mathrm{Na} \mathrm{HPO}_{4}\right.$, $137 \mathrm{mM} \mathrm{NaCl}, \mathrm{pH}$ 7.4) via the proximal epididymal branch until the tissue was cleared of blood (Stoffel et al. 1990a, b). Tissue samples of about $1 \mathrm{~cm}^{3}$ from corresponding epididymal regions were excised, minced with a razor blade and transferred to a sperm washing solution $(6 \% \mathrm{w} / \mathrm{v}$ D-glucose, $0.37 \% \mathrm{w} / \mathrm{v}$ trisodium dihydrate, $0.37 \% \mathrm{w} / \mathrm{v}$ disodium EDTA, $0.12 \% \mathrm{w} / \mathrm{v}$ sodium hydrogen carbonate, $\mathrm{pH}$ 7.2). Minced tissue samples were allowed to liberate sperm cells into the supernatant for 30-60 $\mathrm{min}$.

\section{Ejaculated sperm}

Ejaculates were collected with an artificial vagina at a boar station providing boar semen for artificial insemination (SUISAG, Knutwil, Switzerland) and the sperm-rich fraction was suspended in an appropriate extender (modified after Pursel and Johnson 1976) for transport.

\section{Methods}

\section{Washing and fixation of sperm cells}

Upon arrival in the laboratory, samples were pelleted and washed three times in above-mentioned washing solution with intervening centrifugation $(180 \mathrm{~g}$ for $15 \mathrm{~min})$. The final sperm pellet was resuspended in $1 \mathrm{ml}$ PBS. Sperm cells were fixed in $1 \%$ paraformaldehyde and $1 \%$ glutaraldehyde in $0.2 \mathrm{M}$ cacodylate buffer $(\mathrm{pH}$ 7.4) for $1 \mathrm{~h}$ at room temperature. After thorough washing, sperm cells were incubated in $0.2 \mathrm{M}$ glycine in 0.1 M PBS ( $\mathrm{pH} 7.2$ ) at $4^{\circ} \mathrm{C}$ overnight in order to block the negative charges of free aldehyde residues (Torihara et al. 1995)

\section{Labelling with colloidal gold at pH 2.5}

Labelling was performed as reported previously (Stoffel and Friess 2002). Prior to proceeding with gold labelling, all samples were resuspended in 0.05 M TRIS ( $\mathrm{pH} 2.5$ ). Samples were then incubated with $15 \mathrm{~nm}$ cationic gold (British BioCell International, Brunschwig, Basel, Switzerland) at a dilution of 1:50 in $0.05 \mathrm{M}$ TRIS ( $\mathrm{pH} 2.5$ ) for $2 \mathrm{~h}$ at room temperature and overnight at $4^{\circ} \mathrm{C}$ (Skutelsky and Roth 1986; Skutelsky et al. 1995). After two more washing steps, sperm cells were adsorbed onto gold-sputtered coverslips which had been precoated with poly-L-lysine.

\section{Controls}

Control samples were preincubated with $1 \mathrm{mg} / \mathrm{ml}$ poly-L-lysine in $0.05 \mathrm{M}$ TRIS ( $\mathrm{pH} \mathrm{2.5)}$ ) for $1 \mathrm{~h}$ at room temperature prior to cationic gold labelling.

\section{Processing for SEM}

Samples were osmicated in $1.33 \% \mathrm{OsO}_{4}$ with $0.11 \%$ ruthenium red in 0.13 M cacodylate buffer (pH 7.4; Stoffel et al. 1993), dehydrated through an ascending alcohol series and critical-point dried in a CPD 030 (BAL-TEC, Balzers Liechtenstein) or dried by evaporation of hexamethyldisilazane (Sigma, Buchs, Switzerland; Braet et al. 1997; Ting-Beall et al. 1995). Secondary electron and corresponding backscattered electron images were obtained with the fully digital scanning electron microscope DSM 982 Gemini (Leo, Oberkochen, Germany) at an accelerating voltage of $5 \mathrm{kV}$, a working distance of $6 \mathrm{~mm}$ and a magnification of $10,000 \times$. Individual sperm cells were photographed at random and archived as digital image files.

\section{Labelling counts}

A template was generated in Corel Draw and was overlaid over the respective digital image files (Fig. 1a, b). Parting from the observation that no variation in labelling density was detectable within a given plasma membrane domain (Fig. 1b, e, f), the template used for the counts focused on two wide strips along the midline so as to avoid counts in optically foreshortened membrane areas along the edges. In order to account for minor size variations in randomly sampled sperm cells, counting fields for the respective plasma membrane domains were spaced accordingly. For each of the three membrane domains on every sperm cell, i.e. anterior acrosomal segment, equatorial segment and postacrosomal segment (Fig. 1a), the number of cationised gold particles was determined in four rectangular fields adding up to $0.75 \mu \mathrm{m}^{2}$ (Fig. 1b). Four predefined field combinations to be used for the counts were assigned to individual sperm cells on a sequential basis. Thus, all fields were used equally often. The counts obtained per $0.75 \mu \mathrm{m}^{2}$ ranged from 19 to 469 . Counts were performed on 20-22 sperm cells from every epididymal region and from the ejaculate. Original counts were used for statistical analyses but values obtained were then adjusted to figures giving number of cationised gold particles $/ \mu \mathrm{m}^{2}$.

\section{Statistical analysis}

Preliminary analyses of the labelling data indicated skewed distributions. Therefore, median values and quartiles were used for the descriptive procedures and log transformed data for the analytical procedures. Differences in labelling counts between the six epididymal regions and between the three sperm membrane domains were analysed using a linear model with the log count as the outcome variable. The explanatory variables included the epididymal regions and the sperm membrane domains as fixed effects and, since spermatozoa were sampled at random from a larger population, the sperm cell number as a random effect. In order to analyse differences in the shape of the distributions of labelling counts across epididymal regions, the interaction term of "region times domain" was additionally included and analysed in the model.

The linear model used in the study was defined as follows:

$$
\begin{aligned}
\log (\text { COUNT })= & \text { SPERM }+ \text { REGION }+ \text { DOMAIN } \\
& + \text { REGION } * \text { DOMAIN }
\end{aligned}
$$

where $\log (C O U N T)$ is the natural logarithm of the number of cationised gold particles, SPERM is the number of sperm (1-22), defined as random effect, REGION is the epididymal region (six levels), DOMAIN is the membrane domain (three levels) and REGION*DOMAIN is the first-order interaction term of REGION and DOMAIN. Differences between regions were assessed as based on differences between least square means. Agreement between observed data and model was assessed using residual analysis and evaluation of the variation of the target variable accounted for by the model (R-square values). All statistical procedures were performed using SAS 8.01 (SAS Institute, Cary, N.C., USA) and the level of significance was set at 0.05 throughout the study. 

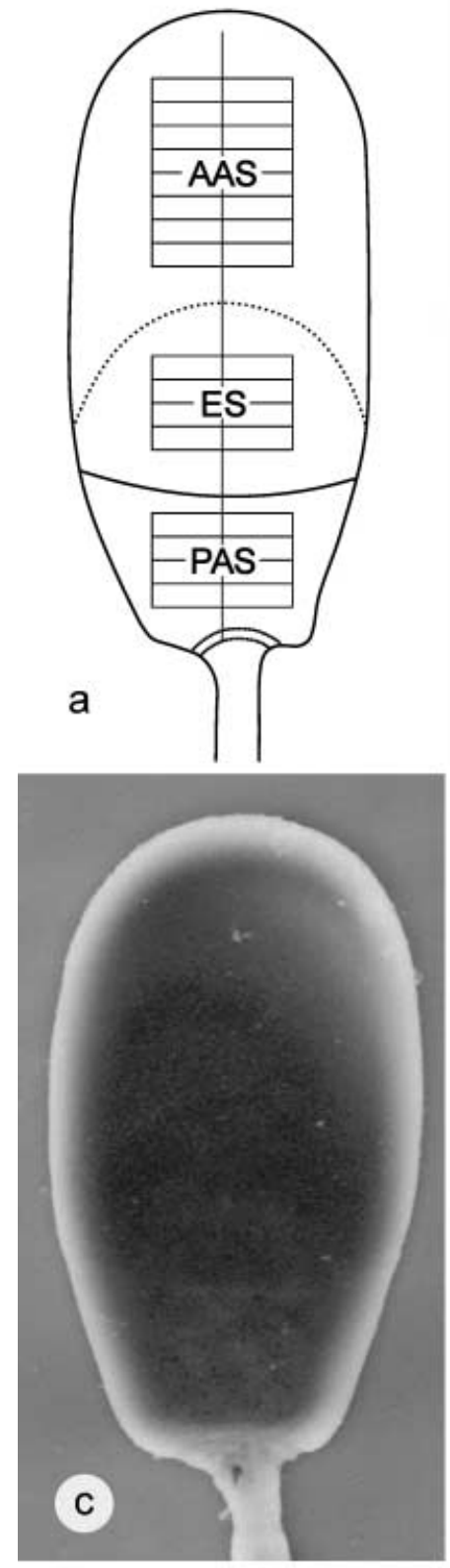

b
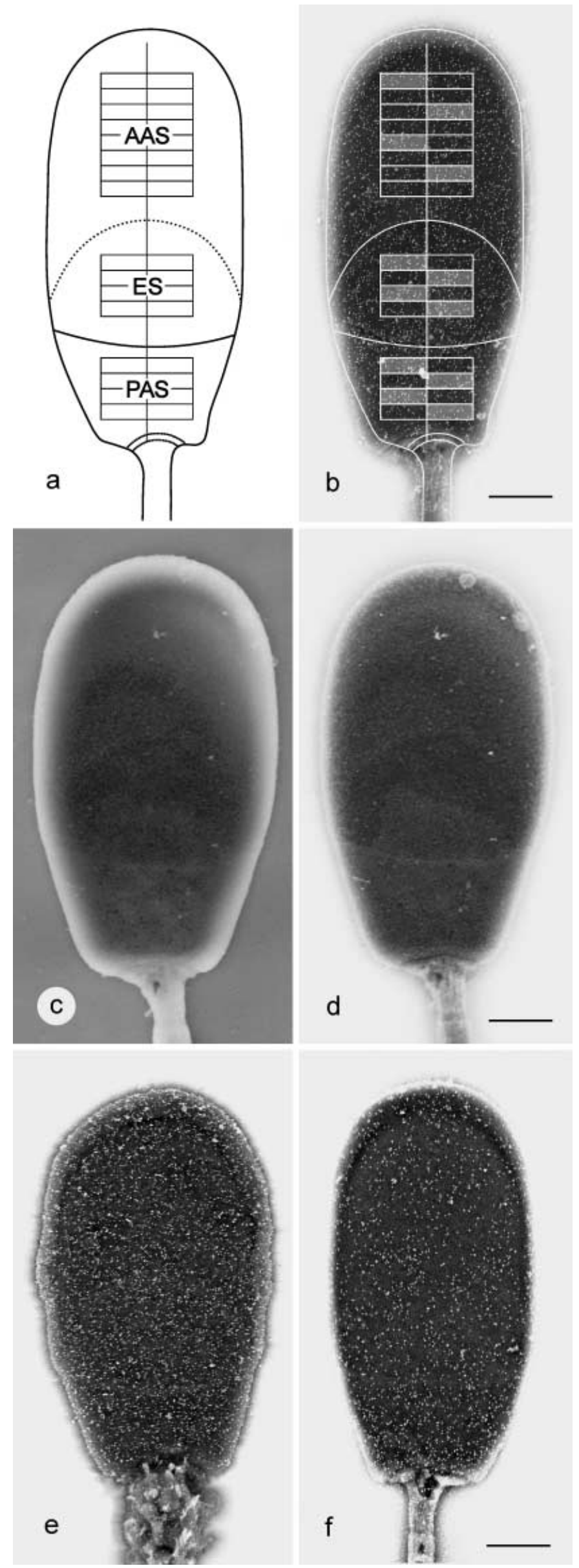

\section{Results}

Control samples were devoid of cationised gold particles (Fig. 1c, d). Consequently, no counts were carried out on the corresponding sperm cells. On labelled spermatozoa, distribution of gold particles was homogenous within each of the three sperm membrane domains (Fig. 1b, e, f). The difference between high and low labelling densities can be perceived qualitatively when extremes are being compared as is shown for efferent duct and proximal corpus spermatozoa, respectively (Fig. 1e, f). Proper assessment of more subtle variations, however, requires a quantitative approach followed by a statistical analysis in order to allow a truthful interpretation of occurring changes in labelling densities.

The explanatory model chosen accounted for $54.6 \%$ of the variation of $\log$ counts, residuals appeared to be normally distributed and no obvious outliers were detected. Median values of labelling counts $/ \mu^{2}$ with corresponding $25 \%$ and $75 \%$ quartiles are given for ejaculated and epididymal spermatozoa (Fig. 2). Labelling density was highest on efferent duct spermatozoa, declined up to the proximal corpus and then sharply increased again. Density of anionic sites on sperm cells from distal corpus and proximal cauda as well as on ejaculated spermatozoa was similar to the level found for proximal caput sperm cells but still was significantly below the values obtained for efferent duct spermatozoa. The $P$ values showed that differences in labelling densities for adjacent epididymal regions were highly significant $(P<0.001)$ except for the comparison between distal corpus and proximal cauda $(P=0.2818)$. All three sperm membrane domains studied underwent similar changes during epididymal transit. Whereas the average density of negative charges on the postacrosomal segment was significantly higher as compared to the acrosomal plasmalemma, no statistical difference in labelling intensities between anterior acrosomal and equatorial segments materialised.

Fig. 1a-f Labelling counts were carried out separately for the three distinct membrane domains on sperm heads, i.e. the anterior acrosomal segment $(A A S)$, the equatorial segment $(E S)$ and the postacrosomal segment $(P A S)$. Cationised gold particles were counted using a template (a) which was overlaid over respective backscattered electron images (b). For each membrane domain, the number of gold particles was determined within four rectangular fields adding up to a surface area of $0.75 \mu \mathrm{m}^{2}(\mathbf{b})$. Predefined combinations of rectangular fields were assigned at random and were used equally often. Counts on control samples were obsolete given that corresponding sperm cells were devoid of labelling (c, $\mathbf{d})$. Distribution of cationised gold particles was homogeneous within each of the three membrane domains considered. This holds true for both heavily labelled efferent duct spermatozoa (e) and for proximal corpus spermatozoa carrying fewer negative charges (f). Intactness of plasmalemma was assessed on secondary electron micrographs (c) whereas labelling counts were performed on backscattered electron micrographs $(\mathbf{b}, \mathbf{d}-\mathbf{f})$. Bars $1 \mu \mathrm{m}$ 


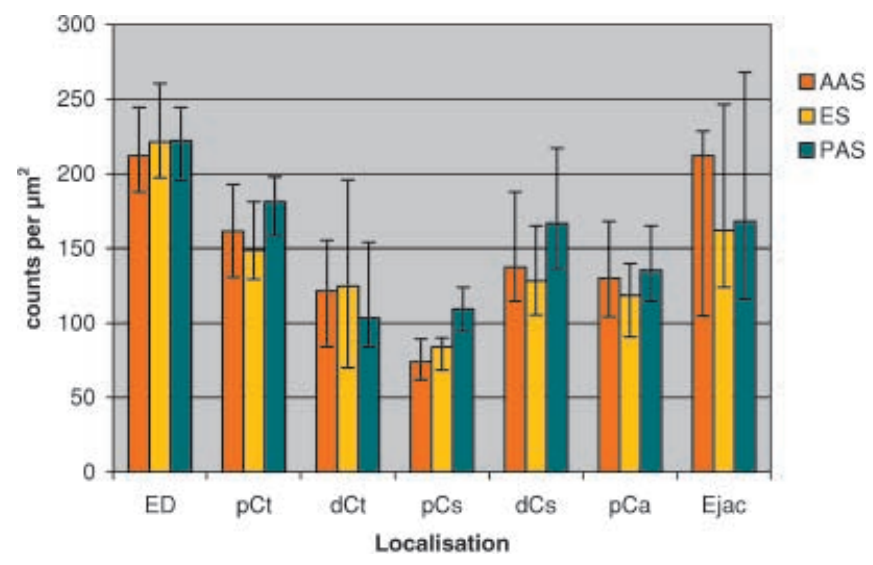

Fig. 2 Labelling counts $/ \mu \mathrm{m}^{2}$ : median values, $25 \%$ and $75 \%$ quartiles are given for the three membrane domains on sperm cells from six epididymal regions as well as for ejaculated spermatozoa. $E D$ Efferent ductules, $p C t$ proximal caput, $d C t$ distal caput, $p C s$ proximal corpus, $d C s$ distal corpus, $p C a$ proximal cauda, Ejac ejaculate, $A A S$ anterior acrosomal segment, $E S$ equatorial segment, $P A S$ postacrosomal segment

\section{Discussion}

With the present study, we demonstrate that visualisation of anionic sites at the sperm surface in SEM after labelling with cationised colloidal gold (Stoffel and Friess 2002) is amenable to quantitative analysis. This approach enabled us to track changes in negative charge distribution within distinct head membrane domains of maturing and ejaculated spermatozoa numerically.

Several factors were carefully monitored in order to ensure the soundness of the labelling counts. Specificity was demonstrated by the virtual absence of any gold particles on the control samples. Furthermore, microscope settings were kept constant throughout the study and the flattened shape of boar spermatozoa ensured their orientation on a coverslip to be perfectly level, this allowing orthogonal visualisation in SEM without any optical foreshortening. Lastly, sperm cells were sampled at random and counts were carried out on a wide area being representative for the respective sperm membrane domains. Thus, the backscattered electron micrographs used were fully standardised and allowed accurate determination of labelling densities as counts per unit surface area. Yet, agreement between observed data and our explanatory model was not absolute. No one-to-one equivalence between the effective number of anionic sites and the labelling counts may be expected and we suggest, therefore, that variations in labelling efficiency from sperm to sperm contributed to some extent to the dispersion of the data collected. Notwithstanding, statistical analysis unambiguously demonstrated the dependability of our labelling protocol and showed that variations in labelling densities observed reflect factual differences in the mean number of negative charges present on sperm cells obtained from the diverse epididymal regions and between sperm membrane domains, respectively. There- fore, the present study provides statistical evidence that our detection system is reliable and that it suits numerical assessment of negative charges at the sperm surface.

As revealed by the least square means, the overall density of negative charges is significantly higher on the postacrosomal segment as compared to the acrosomal plasmalemma. Furthermore, the fact that the "region times domain" interaction did not disclose significant variations indicates that differences between plasmalemmal domains remained constant across the various epididymal regions. Differences in labelling densities between sperm head membrane domains have been reported previously and are suspected to be closely related to specific functional properties. On stallion spermatozoa (Lopez et al. 1987), however, intensity of cationic iron hydroxide binding was estimated to be higher on the acrosomal plasmalemma than in the postacrosomal domain. This discrepancy may reflect species differences. However, the present report is the first to provide quantitative data on differences in labelling densities and in order to substantiate interspecies disparities objectively and reliably, methods allowing statistical analysis should be used to ensure comparability.

As concluded from microelectrophoresis experiments and labelling with cationised ferritin, previous studies contended that the number of negative charges at the sperm surface continuously increases from caput to cauda epididymidis. This is at variance with the results presented herewith which show that labelling density on boar sperm cells first decreases from efferent ductules up to the proximal corpus and then sharply rises again to return to the values observed on proximal caput sperm, all three sperm membrane domains undergoing parallel changes. Differences in labelling densities between adjacent epididymal regions were highly significant from efferent ductules to distal corpus. Values obtained for distal corpus and proximal cauda sperm, however, were statistically equivalent, thus revealing that labelling density stabilises on a level significantly below the numbers obtained for efferent duct spermatozoa. A number of mechanisms have been suggested to account for these changes in density of negative charges such as modifications, removal or masking of pre-existing surface components or, conversely, insertions, unmasking or adsorption of new surface macromolecules (Jones 1989; Voglmayr et al. 1980). But although the range of potential anionic groups involved has been narrowed down in several species (Delgado et al. 1990; Fléchon and Morstin 1975; Holt 1980; Lopez et al. 1987; Moore 1979; Retamal et al. 2000; Schröter et al. 1999; Stoffel and Friess 2002; Toshimori et al. 1988; Yanagimachi et al. 1972), the processes underlying these sperm surface modifications still await definitive elucidation. Irrespective of this gap in current knowledge, our results unequivocally demonstrate that the epididymal environment effectively modulates the density of negative charges at the sperm surface and that anionic sites are not acquired in a linear way along the epididymis but rather are being lost during transit from efferent ducts to proximal corpus and then 
briskly increase in number to reach their final density in the distal corpus. This coincides well with the fact that the distal corpus is the region where boar spermatozoa have attained their full fertilising ability and their potential for motility (Holtz and Smidt 1976; Orgebin-Crist and Olson 1984). The present study, therefore, provides further support for the contention that net negative surface charge is indicative of sperm maturity (Bedford 1963).

Taken together, these findings provide compelling evidence that specific changes in the number of negative surface charges are intimately associated with remodelling of the sperm surface during epididymal transit and thus rightly may be considered to constitute an integral part of the sperm maturation process, a contention which is further supported by recent studies emphasising the importance of charge interactions during fertilisation (Iqbal and Hunter 1995; Kim et al. 1997; Philip et al. 1997; Skutelsky et al. 1992). We, therefore, consider the results presented herewith to provide evidence that changes in the density of negative charges at the sperm surface during epididymal transit result from a precisely orchestrated process which warrants further attention as an important facet of sperm maturation.

Acknowledgements We thank the staff members at SUISAG (Knutwil, Switzerland), who kindly provided boar ejaculates whenever needed. We also are indebted to C. Furer, C. Hug, S. König and $\mathrm{P}$. Thomi for their technical and secretarial assistance.

\section{References}

Bedford JM (1963) Changes in the electrophoretic properties of rabbit spermatozoa during passage through the epididymis. Nature 200:1178-1180

Braet F, Dezanger R, Wisse E (1997) Drying cells for SEM, AFM and TEM by hexamethyldisilazane: a study on hepatic endothelial cells. J Microsc 186:84-87

Chulavatnatol M, Eksittikul T, Toowicharanont P (1978). Control of epididymal sperm motility and approach to male fertility regulation. Int J Androl Suppl 2:629-638

Danon D, Goldstein L, Marikovsky Y, Skutelsky E (1972) Use of cationized ferritin as a label of negative charges on cell surfaces. J Ultrastruct Res 38:500-510

Delgado NM, Reyes R, Carranco A, Huacuja L, Merchant H, Rosado A (1990) Glycosaminoglycan-sulfate as plasma membrane component of pig spermatozoa. Arch Androl 25:121129

Fléchon J-E (1975) Ultrastructural and cytochemical modifications of rabbit spermatozoa during epididymal transport. In: Hafez ESE, Thibault CG (eds) The biology of spermatozoa. Transport, survival and fertilizing ability. Karger, Basel, pp 36-45

Fléchon J-E, Morstin J (1975) Localization of glycoproteins and of negative and positive charges in the cell coat of ejaculated rabbit and bull spermatozoa. Ann Histochim 20:291-300

Holt WV (1980) Surface-bound sialic acid on ram and bull spermatozoa: deposition during epididymal transit and stability during washing. Biol Reprod 23:847-857

Holtz W, Smidt D (1976) The fertilizing capacity of epididymal spermatozoa in the pig. J Reprod Fertil 46:227-229

Iqbal N, Hunter AG (1995) Comparison of various bovine sperm capacitation systems for their ability to alter the net negative surface charge of spermatozoa. J Dairy Sci 78:84-90
Jones R (1989) Membrane remodelling during sperm maturation in the epididymis. In: Milligan SR (ed) Oxford reviews of reproductive biology, vol 11. Oxford University Press, Oxford, pp 285-337

Kim JG, Rock JA, Murphy AA, Parthasarathy S (1997) Chargebased interactions of mammalian sperm with oocytes: inhibition of fertilization of mouse oocytes by ligands of macrophage scavenger receptor(s). Fertil Steril 68:1108-1113

Lopez ML, Souza W de, Bustos-Obregon E (1987) Cytochemical analysis of the anionic sites on the membrane of the stallion spermatozoa during the epididymal transit. Gamete Res 18:319-332

Moore HDM (1979) The net surface charge of mammalian spermatozoa as determined by isoelectric focusing. Changes following sperm maturation, ejaculation, incubation in the female tract, and after enzyme treatment. Int J Androl 2:449-462

Nevo AC, Michaeli I, Schindler H (1961). Electrophoretic properties of bull and of rabbit spermatozoa. Exp Cell Res 23:69-83

Orgebin-Crist M-C, Olson GE (1984) Epididymal sperm maturation. In: Courot $\mathrm{M}$ (ed) The male in farm animal reproduction. Nijhoff, Amsterdam, pp 80-102

Philip J, Rodriguez LG, Bada RA, Ambroise FG, Hernandez UA, Oppenheimer SB (1997) Charge interactions in sperm-egg recognition. Acta Histochem 99:401-410

Pursel VG, Johnson LA (1976) Frozen boar spermatozoa: methods of thawing pellets. J Anim Sci 42:927-931

Retamal C, Urzua J, Lorca C, Lopez ML, Alves EW (2000) Changes in the plasma membrane proteins of stallion spermatozoa during maturation in the epididymis. J Submicrosc Cytol Pathol 32:229-239

Schröter S, Osterhoff C, McArdle W, Ivell R (1999) The Glycocalyx of the sperm surface. Hum Reprod Update 5:302-313

Skutelsky E, Roth J (1986) Cationic colloidal gold: a new probe for the detection of anionic cell surface sites by electron microscopy. J Histochem Cytochem 34:693-696

Skutelsky E, Maymon BB, Maymon R, Shalgi R (1992) Histochemical characterization of anionic constituents in oocytecumulus complex of rats. Histochemistry 98:299-304

Skutelsky E, Shoichetman T, Hammel I (1995) An histochemical approach to characterization of anionic constituents in mast cell secretory granules. Histochem Cell Biol 104:453-458

Stoffel MH, Friess AE (2002) Demonstration and cytochemical analysis of anionic sites on ejaculated boar spermatozoa: a scanning electron microscopy study using cationized colloidal gold. Histochem Cell Biol 117:61-67

Stoffel MH, Friess AE, Kohler T (1990a) Die Vaskularisation des Ebernebenhodens unter besonderer Berücksichtigung der Perfusionsfixation. Schw Arch Tierheilkd 132:571-579

Stoffel MH, Kohler T, Friess AE, Zimmermann W (1990b). Microvasculature of the epididymis in the boar. Cell Tissue Res 259:495-501

Stoffel MH, Frethem C, Hamilton DW, Friess AE (1993) Improved preservation of rat epididymal sperm for high-resolution scanning electron microscopy. Mol Reprod Dev 34:175-182

Ting-Beall HP, Zhelev DV, Hochmuth RM (1995) Comparison of different drying procedures for scanning electron microscopy using human leukocytes. Microsc Res Tech 32:357-361

Toowicharanont P, Chulavatnatol M (1983) Measurement of anionic sites of rat epididymal spermatozoa using tritiated polycationized ferritin. J Reprod Fertil 69:303-306

Torihara K, Morimitsu T, Suganuma T (1995) Anionic sites on Reissner's membrane, stria vascularis, and spiral prominence. J Histochem Cytochem 43:299-305

Toshimori K, Araki S, Oura C (1988) Masking of sperm maturation antigen by sialic acid in the epididymis of the mouse. An immunohistochemical study. Histochemistry 90:195-200

Voglmayr JK, Fairbanks G, Jackowitz MA, Colella JR (1980) Post-testicular developmental changes in the ram sperm cell surface and their relationship to luminal fluid proteins of the reproductive tract. Biol Reprod 22:655-667

Yanagimachi R, Noda YD, Fujimoto M, Nicolson GL (1972) The distribution of negative surface charges on mammalian spermatozoa. Am J Anat 135:497-520 